Article

\title{
Constructing Some Logical Algebras with Hoops
}

\author{
M. Aaly Kologani ${ }^{1}$, Seok-Zun Song ${ }^{2, *} \mathbb{D}$, R. A. Borzooei ${ }^{3} \mathbb{D}$ and Young Bae Jun ${ }^{3,4}$ \\ 1 Hatef Higher Education Institute, Zahedan 9816848165, Iran; mona4011@gmail.com \\ 2 Department of Mathematics, Jeju National University, Jeju 63243, Korea \\ 3 Department of Mathematics, Shahid Beheshti University, Tehran 1983969411, Iran; borzooei@sbu.ac.ir \\ (R.A.B.); skywine@gmail.com (Y.B.J.) \\ 4 Department of Mathematics Education, Gyeongsang National University, Jinju 52828, Korea \\ * Correspondence: szsong@jejunu.ac.kr
}

Received: 12 November 2019; Accepted: 13 December 2019; Published: 16 December 2019

check for updates

\begin{abstract}
In any logical algebraic structures, by using of different kinds of filters, one can construct various kinds of other logical algebraic structures. With this inspirations, in this paper by considering a hoop algebra or a hoop, that is introduced by Bosbach, the notion of co-filter on hoops is introduced and related properties are investigated. Then by using of co-filter, a congruence relation on hoops is defined, and the associated quotient structure is studied. Thus Brouwerian semilattices, Heyting algebras, Wajsberg hoops, Hilbert algebras and BL-algebras are obtained.
\end{abstract}

Keywords: hoop; co-filter; Brouwerian semilattice; Heyting algebra; Wajsberg hoop; Hilbert algebra; BL-algebra

MSC: 03G99; 06B10

\section{Introduction}

Non-classical logics (or called alternative logics) are formal systems that differ in a significant way from standard logical systems such as propositional and predicate logic. Many-valued logics are non-classical logics which are similar to classical logic. Bosbach [1,2] proposed the concept of hoop which is a nice algebraic structure to research the many-valued logical system whose propositional value is given in a lattice. For various information on hoops, refer to [3-8].

In this paper, we introduce the notion of co-filter in hoops and we get some properties of it. Then we construct a congruence relation by using co-filters on hoops. Finally, we investigate under which conditions the quotient structure of this congruence relation will be Brouwerian semilattice, Heyting algebra, Wajsberg hoop, Hilbert algebra and BL-algebra.

\section{Preliminaries}

In this section, we recollect some definitions and results which will be used in the following and we shall not cite them every time they are used.

Definition 1 ([9]). A hoop is an algebraic structure $(\mathbb{H}, \odot, \rightarrow, 1)$ of type $(2,2,0)$ such that, for all $\alpha, \beta, \gamma \in \mathbb{H}$ it satisfies in the following conditions:

$(H P 1) \quad(\mathbb{H}, \odot, 1)$ is a commutative monoid.

(HP2) $\alpha \rightarrow \alpha=1$.

$($ HP3 $)(\alpha \odot \beta) \rightarrow \gamma=\alpha \rightarrow(\beta \rightarrow \gamma)$.

$(H P 4) \quad \alpha \odot(\alpha \rightarrow \beta)=\beta \odot(\beta \rightarrow \alpha)$. 
On hoop $\mathbb{H}$, a binary relation $\leq$ is defined on $\mathbb{H}$ such that $\alpha \leq \beta$ iff $\alpha \rightarrow \beta=1$ and $(\mathbb{H}, \leq)$ is a poset. If the least element $0 \in \mathbb{H}$ exists such that, for all $\alpha \in \mathbb{H}, 0 \leq \alpha$, then $\mathbb{H}$ is called a bounded hoop. We let $\alpha^{0}=1$ and $\alpha^{n}=\alpha^{n-1} \odot \alpha$, for any $n \in \mathbb{N}$. If $\mathbb{H}$ is bounded, then, for all $\alpha \in \mathbb{H}$, the operation negation " 1 "is defined on $\mathbb{H}$ by, $\alpha^{\prime}=\alpha \rightarrow 0$. If $\left(\alpha^{\prime}\right)^{\prime}=\alpha$, for all $\alpha \in \mathbb{H}$, then $\mathbb{H}$ is said to have (DNP) property.

Proposition 1 ([1,2]). Let $(\mathbb{H}, \odot, \rightarrow, 1)$ be a hoop. Then, for all $\alpha, \beta, \gamma \in \mathbb{H}$, it satisfies in the following conditions:

(i) $(\mathbb{H}, \leq)$ is a meet-semilattice with $\alpha \wedge \beta=\alpha \odot(\alpha \rightarrow \beta)$.

(ii) $\alpha \odot \beta \leq \gamma$ iff $\alpha \leq \beta \rightarrow \gamma$.

(iii) $\alpha \odot \beta \leq \alpha, \beta$ and $\alpha^{n} \leq \alpha$, for any $n \in \mathbb{N}$.

(iv) $\alpha \leq \beta \rightarrow \alpha$.

(v) $1 \rightarrow \alpha=\alpha$ and $\alpha \rightarrow 1=1$.

(vi) $\alpha \odot(\alpha \rightarrow \beta) \leq \beta$.

(vii) $\alpha \rightarrow \beta \leq(\beta \rightarrow \gamma) \rightarrow(\alpha \rightarrow \gamma)$.

(viii) $\alpha \leq \beta$ implies $\alpha \odot \gamma \leq \beta \odot \gamma, \gamma \rightarrow \alpha \leq \gamma \rightarrow \beta$ and $\beta \rightarrow \gamma \leq \alpha \rightarrow \gamma$.

Proposition 2 ([1,2]). Let $\mathbb{H}$ be a bounded hoop. Then, for any $\alpha, \beta \in \mathbb{H}$, the following conditions hold:

(i) $\alpha \leq \alpha^{\prime \prime}$ and $\alpha \odot \alpha^{\prime}=0$

(ii) $\alpha^{\prime} \leq \alpha \rightarrow \beta$.

(iii) $\alpha^{\prime \prime \prime}=\alpha^{\prime}$.

(iv) If $\mathbb{H}$ has (DNP), then $\alpha \rightarrow \beta=\beta^{\prime} \rightarrow \alpha^{\prime}$.

(v) If $\mathbb{H}$ has (DNP), then $(\alpha \rightarrow \beta) \rightarrow \beta=(\beta \rightarrow \alpha) \rightarrow \alpha$.

Proposition 3 ([10]). Let $\mathbb{H}$ be a hoop and for any $\alpha, \beta \in \mathbb{H}$, define the operation $\vee$ on $\mathbb{H}$ as follows,

$$
\alpha \vee \beta=((\alpha \rightarrow \beta) \rightarrow \beta) \wedge((\beta \rightarrow \alpha) \rightarrow \alpha)
$$

Then, for all $\alpha, \beta, \gamma \in \mathbb{H}$, the following conditions are equivalent:

(i) $\vee$ is associative,

(ii) $\alpha \leq \beta$ implies $\alpha \vee \gamma \leq \beta \vee \gamma$,

(iii) $\alpha \vee(\beta \wedge \gamma) \leq(\alpha \vee \beta) \wedge(\alpha \vee \gamma)$,

(iv) $\vee$ is the join operation on $\mathbb{H}$.

A hoop $\mathbb{H}$ is said to $a \vee$-hoop, if it satisfies one of the above equivalent conditions.

Proposition 4 ([10]). Let $\mathbb{H}$ be $a \vee$-hoop and $\alpha, \beta, \gamma \in \mathbb{H}$. Then $\vee$-hoop $(\mathbb{H}, \vee, \wedge)$ is a distributive lattice and $(\alpha \vee \beta) \rightarrow \gamma=(\alpha \rightarrow \gamma) \wedge(\beta \rightarrow \gamma)$

Definition 2 ([10]). A non-empty subset $F$ of a hoop $\mathbb{H}$ is called a filter of $\mathbb{H}$ if, for any $\alpha, \beta \in \mathbb{H}$, the following condition hold:

(F1) $\alpha, \beta \in F$ implies $\alpha \odot \beta \in F$.

(F2) $\alpha \leq \beta$ and $\alpha \in F$ imply $\beta \in F$.

The set of all filters of $\mathbb{H}$ is denoted by $\mathcal{F}(\mathbb{H})$. Clearly, for any filter $F$ of $\mathbb{H}, 1 \in F$. F is called a proper filter if $F \neq \mathbb{H}$. So, if $\mathbb{H}$ is a bounded hoop, then a filter is proper iff it does not contain 0 . It is easy to see that $F \in \mathcal{F}(\mathbb{H})$ iff, for any $\alpha, \beta \in \mathbb{H}, 1 \in F$ and if $\alpha, \alpha \rightarrow \beta \in F$, then $\beta \in F$.

\section{Co-Filters in Hoops}

From here on, if there is no mention, $\mathbb{H}$ denotes a bounded hoop.

We introduce the notion of co-filters on hoops, and it is proved that co-filters are not filters and some properties of them are studied. Moreover, a congruence relation is defined by them and is investigated the quotient structure of this congruence relation. 
Definition 3. A subset I of $\mathbb{H}$ is said to be a co-filter of $\mathbb{H}$ if, for any $\alpha, \beta \in \mathbb{H}$,

$\left(C F_{1}\right) 0 \in I$.

$\left(C F_{2}\right)(\alpha \rightarrow \beta)^{\prime} \in I$ and $\beta \in I$ imply $\alpha \in I$.

Example 1. Let $\mathbb{H}=\{0, a, b, c, d, 1\}$. Define the operations $\odot$ and $\rightarrow$ on $\mathbb{H}$ as below,

\begin{tabular}{l|llllll}
$\rightarrow$ & 0 & $a$ & $b$ & $c$ & $d$ & 1 \\
\hline 0 & 1 & 1 & 1 & 1 & 1 & 1 \\
$a$ & $d$ & 1 & $d$ & 1 & $d$ & 1 \\
$b$ & $c$ & $c$ & 1 & 1 & 1 & 1 \\
$c$ & $b$ & $c$ & $d$ & 1 & $d$ & 1 \\
$d$ & $a$ & $a$ & $b$ & $c$ & 1 & 1 \\
1 & 0 & $a$ & $b$ & $c$ & $d$ & 1
\end{tabular}

\begin{tabular}{l|llllll}
$\odot$ & 0 & $a$ & $b$ & $c$ & $d$ & 1 \\
\hline 0 & 0 & 0 & 0 & 0 & 0 & 0 \\
$a$ & 0 & $a$ & 0 & $a$ & 0 & $a$ \\
$b$ & 0 & 0 & 0 & 0 & $b$ & $b$ \\
$c$ & 0 & $a$ & 0 & $a$ & $b$ & $c$ \\
$d$ & 0 & 0 & $b$ & $b$ & $d$ & $d$ \\
1 & 0 & $a$ & $b$ & $c$ & $d$ & 1
\end{tabular}

Then $(\mathbb{H}, \odot, \rightarrow, 0,1)$ is a hoop and $I=\{0, b, d\}$ is a co-filter of $\mathbb{H}$, which is not a filter of $\mathbb{H}$ because $1 \notin I$.

Note. For $S \subseteq \mathbb{H}$, define $S^{\prime}=\left\{\alpha \in \mathbb{H} \mid \alpha^{\prime} \in S\right\}$

Proposition 5. If $\mathbb{H}$ has (DNP) and $\varnothing \neq I \subseteq \mathbb{H}$, then $I$ is a filter of $\mathbb{H}$ iff $I^{\prime}$ is a co-filter of $\mathbb{H}$.

Proof. $(\Rightarrow)$ Suppose $I \in \mathcal{F}(\mathbb{H})$. Then $1 \in I$, and so $0=1^{\prime} \in I^{\prime}$. Let $\alpha, \beta \in \mathbb{H}$ such that $(\alpha \rightarrow \beta)^{\prime} \in I^{\prime}$ and $\beta \in I^{\prime}$. Since $\mathbb{H}$ has (DNP), by Proposition 2(iv), $\beta^{\prime} \rightarrow \alpha^{\prime}=\alpha \rightarrow \beta \in I, \beta^{\prime} \in I$ and since $I \in \mathcal{F}(\mathbb{H})$, by Definition $2, \alpha^{\prime} \in I$, and so $\alpha^{\prime \prime}=\alpha \in I^{\prime}$. Hence, $I^{\prime}$ is a co-filter of $\mathbb{H}$.

$(\Leftarrow)$ Let $I^{\prime}$ be a co-filter of $\mathbb{H}$. Then $0 \in I^{\prime}$, and so $1 \in I$. Now, suppose $\alpha, \beta \in A$ such that $\alpha, \alpha \rightarrow \beta \in I$. Thus $(\alpha \rightarrow \beta)^{\prime} \in I^{\prime}$ and $\alpha^{\prime} \in I^{\prime}$. Since $\mathbb{H}$ has (DNP), by Proposition 2(iv), $\left(\beta^{\prime} \rightarrow \alpha^{\prime}\right)^{\prime} \in I^{\prime}, \alpha^{\prime} \in I^{\prime}$ and since $I^{\prime}$ is a co-filter of $\mathbb{H}$, by definition, $\beta^{\prime} \in I^{\prime}$. Hemce, by (DNP), $\beta \in I$. Therefore, $I \in \mathcal{F}(\mathbb{H})$.

If $\mathbb{H}$ does not have (DNP), then Proposition 5 is not true, in general. We show this in the following example.

Example 2. Let $\mathbb{H}=\{0, a, b, 1\}$ be a chain such that $0 \leq a \leq b \leq 1$ and two binary operations $\odot$ and $\rightarrow$ which are given below,

\begin{tabular}{l|llll}
$\rightarrow$ & 0 & $a$ & $b$ & 1 \\
\hline 0 & 1 & 1 & 1 & 1 \\
$a$ & $a$ & 1 & 1 & 1 \\
$b$ & 0 & $a$ & 1 & 1 \\
1 & 0 & $a$ & $b$ & 1
\end{tabular}

\begin{tabular}{l|llll}
$\odot$ & 0 & $a$ & $b$ & 1 \\
\hline 0 & 0 & 0 & 0 & 0 \\
$a$ & 0 & 0 & $a$ & $a$ \\
$b$ & 0 & $a$ & $b$ & $b$ \\
1 & 0 & $a$ & $b$ & 1
\end{tabular}

By routine calculations, $(\mathbb{H}, \odot, \rightarrow, 0,1)$ is a hoop that does not have $(D N P)$. It is clear that $\mathbb{H}$ is a co-filter of $\mathbb{H}$ but $\mathbb{H}^{\prime}=\{0, a, 1\}$ is not a filter of $\mathbb{H}$.

Note. If $F$ is a proper filter of $\mathbb{H}$, then by Definition 2, $0 \notin F$. Thus, $F$ is not a co-filter of $\mathbb{H}$. On the other hand, for any proper co-filter $I$ of $\mathbb{H}$, if $1 \notin I$, then $I \notin \mathcal{F}(\mathbb{H})$.

Proposition 6. Let I be a co-filter of $\mathbb{H}$. Then the following statements hold:

(i) If $\alpha \leq \beta$ and $\beta \in I$, then $\alpha \in I$, for any $\alpha, \beta \in \mathbb{H}$.

(ii) If $\alpha \in I$, then $\alpha \odot \beta \in I$, for any $\beta \in \mathbb{H}$.

(iii) If $\mathbb{H}$ is a $\vee$-hoop with (DNP), then $\alpha \vee \beta \in I$, for any $\alpha, \beta \in I$. 
Proof. (i) Let $\alpha, \beta \in \mathbb{H}$ such that $\alpha \leq \beta$ and $\beta \in I$. Then $\alpha \rightarrow \beta=1$, and so $(\alpha \rightarrow \beta)^{\prime}=0 \in I$. Since $I$ is a co-filter of $\mathbb{H},(\alpha \rightarrow \beta)^{\prime} \in I$ and $\beta \in I$, we have $\alpha \in I$.

(ii) Let $\alpha, \beta \in \mathbb{H}$ and $\alpha \in I$. By Proposition 1(iii), $\alpha \odot \beta \leq \alpha$. Since $\alpha \in I$, by (i), $\alpha \odot \beta \in I$.

(iii) Suppose $\alpha, \beta \in I$. By Proposition 4,

$$
(\alpha \vee \beta) \rightarrow \beta=(\alpha \rightarrow \beta) \wedge(\beta \rightarrow \beta)=\alpha \rightarrow \beta,
$$

then $((\alpha \vee \beta) \rightarrow \beta)^{\prime}=(\alpha \rightarrow \beta)^{\prime}$. By Proposition 2(ii), $\alpha^{\prime} \leq \alpha \rightarrow \beta$, and so, by Proposition 1(viii) and (DNP), $(\alpha \rightarrow \beta)^{\prime} \leq \alpha^{\prime \prime}=\alpha$. Hence, $((\alpha \vee \beta) \rightarrow \beta)^{\prime} \leq \alpha$. From $I$ is a co-filter of $\mathbb{H}$ and $\alpha \in I$, by (i) $((\alpha \vee \beta) \rightarrow \beta)^{\prime} \in I$. Moreover, by assumption, $\beta \in I$ and $I$ is a co-filter of $\mathbb{H}$. Therefore, $\alpha \vee \beta \in I$.

Corollary 1. If $I$ is a co-filter of $\mathbb{H}$ and $1 \in I$, then $I=\mathbb{H}$.

Proof. By Proposition 6(i), the proof is straightforward.

We provide conditions for a nonempty subset to be a co-filter.

Proposition 7. Let $\alpha, \beta \in \mathbb{H}$ and $\varnothing \neq I \subseteq \mathbb{H}$ such that I has the following properties,

(i) if $\alpha, \beta \in I$, then $\alpha^{\prime} \rightarrow \beta \in I$,

(ii) if $\alpha \leq \beta$ and $\beta \in I$, then $\alpha \in I$.

Then I is a co-filter of $\mathbb{H}$.

Proof. Let $\alpha \in I$. Since, for all $\alpha \in \mathbb{H}, 0 \leq \alpha$, by (ii), $0 \in I$. Suppose $\alpha, \beta \in \mathbb{H}$ such that $(\alpha \rightarrow \beta)^{\prime} \in I$ and $\beta \in I$. Then by (i),

$$
\beta^{\prime} \rightarrow(\alpha \rightarrow \beta)^{\prime}=\beta^{\prime} \rightarrow((\alpha \rightarrow \beta) \rightarrow 0) \in I .
$$

By (HP3), $(\alpha \rightarrow \beta) \rightarrow \beta^{\prime \prime} \in I$. Moreover, by Propositions 2(i) and 1(viii), $\beta \leq \beta^{\prime \prime}$ and so $(\alpha \rightarrow \beta) \rightarrow$ $\beta \leq(\alpha \rightarrow \beta) \rightarrow \beta^{\prime \prime}$. Since $(\alpha \rightarrow \beta) \rightarrow \beta^{\prime \prime} \in I$, by (ii), $(\alpha \rightarrow \beta) \rightarrow \beta \in I$. Also, by Proposition 1(vi), $\alpha \leq(\alpha \rightarrow \beta) \rightarrow \beta$, and by (ii), $\alpha \in I$. Hence $I$ is a co-filter of $\mathbb{H}$.

By below example, we show that the converse of Proposition 7, is not true.

Example 3. Let $\mathbb{H}=\{0, a, b, c, 1\}$ be a set with the following Cayley tabels:

\begin{tabular}{l|lllll}
$\rightarrow$ & 0 & $a$ & $b$ & $c$ & 1 \\
\hline 0 & 1 & 1 & 1 & 1 & 1 \\
$a$ & $b$ & 1 & 0 & 0 & 1 \\
$b$ & $c$ & 0 & 1 & 0 & 1 \\
$c$ & $c$ & 0 & 0 & 1 & 1 \\
1 & 0 & $a$ & $b$ & $c$ & 1
\end{tabular}

\begin{tabular}{l|lllll}
$\odot$ & 0 & $a$ & $b$ & $c$ & 1 \\
\hline 0 & 0 & 0 & 0 & 0 & 0 \\
$a$ & 0 & $a$ & 0 & 0 & $a$ \\
$b$ & 0 & 0 & $b$ & 0 & $b$ \\
$c$ & 0 & 0 & 0 & $c$ & $c$ \\
1 & 0 & $a$ & $b$ & $c$ & 1
\end{tabular}

Then $(\mathbb{H}, \odot, \rightarrow, 0,1)$ is a hoop and $I=\{0, a\}$ is a co-filter of $\mathbb{H}$ but $a^{\prime} \rightarrow 0=b \rightarrow 0=c \notin I$.

Proposition 8. Let $\mathbb{H}$ has (DNP). Then I is a co-filter of $\mathbb{H}$ iff for any $\alpha, \beta \in \mathbb{H}$, I has the following properties,

(i) if $\alpha, \beta \in I$, then $\alpha^{\prime} \rightarrow \beta \in I$.

(ii) if $\alpha \leq \beta$ and $\beta \in I$, then $\alpha \in I$.

Proof. $(\Rightarrow)$ Let $I$ be a co-filter of $\mathbb{H}$. Then by Proposition 6(i), item (ii) is clear. Suppose $\alpha, \beta \in I$. By Proposition 1(vi) and (viii), $\left(\left(\alpha^{\prime} \rightarrow \beta\right) \rightarrow \beta\right)^{\prime} \leq \alpha^{\prime \prime}$. Since $\mathbb{H}$ has (DNP), $\left(\left(\alpha^{\prime} \rightarrow \beta\right) \rightarrow \beta\right)^{\prime} \leq \alpha$. By assumption, $\alpha \in I$, and so by Proposition 6(i), $\left(\left(\alpha^{\prime} \rightarrow \beta\right) \rightarrow \beta\right)^{\prime} \in I$. Moreover, since $\beta \in I$ and $I$ is a co-filter of $\mathbb{H}, \alpha^{\prime} \rightarrow \beta \in I$.

$(\Leftarrow)$ The proof is similar to the proof of Proposition 7 . 
Theorem 1. Let I be a co-filter of $\mathbb{H}$. Then, for all $\alpha, \beta, \gamma \in \mathbb{H}$, the following statements hold:

(i) $((\alpha \rightarrow \beta) \rightarrow \alpha)^{\prime} \in I$ and $\alpha \in I$ imply $\beta \in I$.

(ii) If $\alpha \rightarrow(\alpha \rightarrow \beta) \in I$, then $\alpha \rightarrow \beta \in I$.

(iii) $((\beta \rightarrow(\beta \rightarrow \alpha)) \rightarrow \gamma)^{\prime} \in$ I and $\gamma \in I$ imply $\beta \rightarrow \alpha \in I$.

(iv) If $(\alpha \rightarrow \beta)^{\prime} \in I$, then $\left((\alpha \rightarrow \beta)^{\prime} \rightarrow \beta\right)^{\prime} \in I$.

Proof. (i) Let $\alpha, \beta \in \mathbb{H}$ such that $((\alpha \rightarrow \beta) \rightarrow \alpha)^{\prime} \in I$ and $\alpha \in I$. Since $I$ is a co-filter of $\mathbb{H}, \alpha \rightarrow \beta \in I$. By Proposition 1(iv), $\beta \leq \alpha \rightarrow \beta \in I$. From Proposition 6(i), $\beta \in I$.

(ii) By Proposition 1(iii) and (viii), $\alpha^{2} \leq \alpha$ and $\alpha \rightarrow \beta \leq \alpha^{2} \rightarrow \beta=\alpha \rightarrow(\alpha \rightarrow \beta)$. Since $\alpha \rightarrow(\alpha \rightarrow$ $\beta) \in I$, by Proposition 6(i), $\alpha \rightarrow \beta \in I$.

(iii) Suppose $\alpha, \beta, \gamma \in \mathbb{H}$ such that $((\beta \rightarrow(\beta \rightarrow \alpha)) \rightarrow \gamma)^{\prime} \in I$ and $\gamma \in I$. Since $I$ is a co-filter of $\mathbb{H}$, $\beta \rightarrow(\beta \rightarrow \alpha) \in I$, and so by (ii), $\beta \rightarrow \alpha \in I$.

(iv) Let $\alpha, \beta \in \mathbb{H}$ such that $(\alpha \rightarrow \beta)^{\prime} \in I$. Then by (HP3), we have

$$
\begin{array}{rlrl}
\left((\alpha \rightarrow \beta)^{\prime} \rightarrow \beta\right)^{\prime} \rightarrow(\alpha \rightarrow \beta)^{\prime} & =(\alpha \rightarrow \beta) \rightarrow\left((\alpha \rightarrow \beta)^{\prime} \rightarrow \beta\right)^{\prime \prime} & \text { by Propositions 1(viii) and 2(i) } \\
& \geq(\alpha \rightarrow \beta) \rightarrow\left((\alpha \rightarrow \beta)^{\prime} \rightarrow \beta\right) & \text { by (HP3) } \\
& =\left[(\alpha \rightarrow \beta) \odot(\alpha \rightarrow \beta)^{\prime}\right] \rightarrow \beta \quad \text { by Proposition 2(i) } \\
& =0 \rightarrow \beta \\
& =1
\end{array}
$$

Thus, $\left((\alpha \rightarrow \beta)^{\prime} \rightarrow \beta\right)^{\prime} \rightarrow(\alpha \rightarrow \beta)^{\prime}=1$, and so $\left((\alpha \rightarrow \beta)^{\prime} \rightarrow \beta\right)^{\prime} \leq(\alpha \rightarrow \beta)^{\prime}$. Since $(\alpha \rightarrow \beta)^{\prime} \in I$ and $I$ is a co-filter of $\mathbb{H}$, by Proposition $6(\mathrm{i}),\left((\alpha \rightarrow \beta)^{\prime} \rightarrow \beta\right)^{\prime} \in I$.

If $X \subseteq \mathbb{H}$, then the least co-filter of $\mathbb{H}$ contains $X$ is called the co-filter generated by $X$ of $\mathbb{H}$ and we show it by $[X)$.

Theorem 2. If $\mathbb{H}$ has (DNP), then, for any $a \in \mathbb{H}$,

$$
[a)=\left\{\alpha \in A \mid \exists n \in \mathbb{N} \text { such that }\left(a^{\prime}\right)^{n} \leq \alpha^{\prime}\right\}
$$

Proof. Let $B=\left\{\alpha \in A \mid \exists n \in \mathbb{N}\right.$ such that $\left.\left(a^{\prime}\right)^{n} \leq \alpha^{\prime}\right\}$. Since $\left(a^{\prime}\right)^{n} \leq 1=0^{\prime}$, for all $n \in \mathbb{N}$, we have $0 \in B$, and so $B \neq \varnothing$. Now, suppose $\alpha, \beta \in \mathbb{H}$ such that $(\alpha \rightarrow \beta)^{\prime} \in B$ and $\beta \in B$. Then there exist $n, m \in \mathbb{N}$, such that $\left(a^{\prime}\right)^{n} \leq(\alpha \rightarrow \beta)^{\prime \prime}$ and $\left(a^{\prime}\right)^{m} \leq \beta^{\prime}$. By Proposition 1(viii),

$$
\left(a^{\prime}\right)^{n} \odot\left(a^{\prime}\right)^{m} \leq(\alpha \rightarrow \beta)^{\prime \prime} \odot\left(a^{\prime}\right)^{m} \leq(\alpha \rightarrow \beta)^{\prime \prime} \odot \beta^{\prime}
$$

By (HP3), we get

$$
\begin{aligned}
\left((\alpha \rightarrow \beta)^{\prime \prime} \odot \beta^{\prime}\right) \rightarrow \alpha^{\prime} & =\beta^{\prime} \rightarrow\left((\alpha \rightarrow \beta)^{\prime \prime} \rightarrow \alpha^{\prime}\right) \\
& =\beta^{\prime} \rightarrow\left(\alpha \rightarrow(\alpha \rightarrow \beta)^{\prime \prime \prime}\right) \text { by Proposition 2(iii) } \\
& =\beta^{\prime} \rightarrow\left(\alpha \rightarrow(\alpha \rightarrow \beta)^{\prime}\right) \\
& =\alpha \rightarrow\left(\beta^{\prime} \rightarrow(\alpha \rightarrow \beta)^{\prime}\right) \\
& =\alpha \rightarrow\left((\alpha \rightarrow \beta) \rightarrow \beta^{\prime \prime}\right) \\
& =(\alpha \rightarrow \beta) \rightarrow\left(\alpha \rightarrow \beta^{\prime \prime}\right) \text { by Propositions 2(i) and 1(viii) } \\
& =1
\end{aligned}
$$

Then $(\alpha \rightarrow \beta)^{\prime \prime} \odot \beta^{\prime} \leq \alpha^{\prime}$, and so $\left(a^{\prime}\right)^{n} \odot\left(a^{\prime}\right)^{m} \leq \alpha^{\prime}$. Hence, $n+m \in \mathbb{N}$ exists such that $\left(a^{\prime}\right)^{n+m} \leq \alpha^{\prime}$. Therefore, $\alpha \in B$, and so $B$ is a co-filter of $\mathbb{H}$. Also, by Proposition 1 (iii), $\left(a^{\prime}\right)^{n} \leq a^{\prime}$. Thus, $a \in B$ and $B$ is a co-filter of $\mathbb{H}$ which containing $a$. Now, it is enough to prove that $B$ is the least co-filter of $\mathbb{H}$ which containing $a$. Suppose $C$ is a co-filter of $\mathbb{H}$ that contains $a$. We show that $B \subseteq C$. Let $\alpha \in B$. Then there 
exists $n \in \mathbb{N}$ such that $\left(a^{\prime}\right)^{n} \leq \alpha^{\prime}$. Thus $\left(a^{\prime}\right)^{n} \rightarrow \alpha^{\prime}=1$. Since $\mathbb{H}$ has (DNP), by (HP3) and Proposition 2(iv), we get

$$
\begin{aligned}
1 & =\left(a^{\prime}\right)^{n} \rightarrow \alpha^{\prime} \\
& =\left(\left(a^{\prime}\right)^{n-1} \odot\left(a^{\prime}\right)\right) \rightarrow \alpha^{\prime} \\
& =\left(a^{\prime}\right)^{n-1} \rightarrow\left(a^{\prime} \rightarrow \alpha^{\prime}\right) \\
& =\left(a^{\prime}\right)^{n-1} \rightarrow\left(\alpha \rightarrow a^{\prime \prime}\right) \\
& =\left(a^{\prime}\right)^{n-1} \rightarrow(\alpha \rightarrow a) \\
& =\left(\left(a^{\prime}\right)^{n-2} \odot\left(a^{\prime}\right)\right) \rightarrow(\alpha \rightarrow a) \\
& =\left(a^{\prime}\right)^{n-2} \rightarrow\left(a^{\prime} \rightarrow(\alpha \rightarrow a)\right) \\
& =\left(a^{\prime}\right)^{n-2} \rightarrow\left((\alpha \rightarrow a)^{\prime} \rightarrow a^{\prime \prime}\right) \\
& =\left(a^{\prime}\right)^{n-2} \rightarrow\left((\alpha \rightarrow a)^{\prime} \rightarrow a\right)
\end{aligned}
$$

By continuing this method, we have

$$
1=\left(\left(\left((\alpha \rightarrow a)^{\prime} \rightarrow a\right)^{\prime} \rightarrow a\right)^{\prime} \rightarrow \ldots \rightarrow a\right)^{\prime} \rightarrow a
$$

Hence,

$$
\left.\left.\left.\left[\left(\left(\left((\alpha \rightarrow a)^{\prime} \rightarrow a\right)^{\prime} \rightarrow a\right)^{\prime}\right) \rightarrow\right) \ldots\right) \rightarrow a\right)^{\prime} \rightarrow a\right]^{\prime}=1^{\prime}=0 \in C .
$$

Since $C$ is a co-filter of $\mathbb{H}$ and $a \in C$, we obtain,

$$
\left.\left.\left(\left(\left(\left((\alpha \rightarrow a)^{\prime} \rightarrow a\right)^{\prime} \rightarrow a\right)^{\prime}\right) \rightarrow\right) \ldots\right) \rightarrow a\right)^{\prime} \in C .
$$

By continuing this method, we can see that $(\alpha \rightarrow a)^{\prime} \in C$. Since $(\alpha \rightarrow a)^{\prime} \in C, a \in C$ and $C$ is a co-filter of $\mathbb{H}$, we have $\alpha \in C$. Hence, $B \subseteq C$. Therefore, $B=[a)$.

Corollary 2. Let $\mathbb{H}$ has (DNP), $X \subseteq \mathbb{H}$ and $a \in \mathbb{H}$. Then the following statements hold:

(i) $[X)=\left\{\alpha \in A \mid \exists n \in \mathbb{N}\right.$ and $a_{1}, \ldots, a_{n} \in X$ s.t, $\left.a_{1}{ }^{\prime} \odot a_{2}{ }^{\prime} \odot \ldots \odot a_{n}{ }^{\prime} \leq \alpha^{\prime}\right\}$.

(ii) $[I \cup\{a\})=\left\{\beta \in A \mid \exists n, m \in \mathbb{N}\right.$ and $\alpha_{1}, \ldots, \alpha_{m} \in I$ s.t, $\left.\left(\alpha_{1}^{\prime} \odot \ldots \odot \alpha_{m}^{\prime}\right) \odot\left(a^{\prime}\right)^{n} \leq \beta^{\prime}\right\}$.

Proof. The proof is similar to the proof of Theorem 2.

Example 4. Let $A$ be the hoop as in Example 3. It is clear that $A$ has (DNP). Since $a^{\prime}=d$ and $d \leq d=a^{\prime}$ and $d \leq 1=0^{\prime}$, we get $[a)=\{0, a\}$. Also, since $d^{\prime}=a$ and $a \leq 1, a, c$, we have $[d)=\{0, b, d\}$.

Theorem 3. Let I be a co-filter of $\mathbb{H}$. We define the relation $\equiv_{I}$ on $\mathbb{H}$ as follows,

$$
\alpha \equiv_{I} \beta \text { iff }(\alpha \rightarrow \beta)^{\prime} \in \text { I and }(\beta \rightarrow \alpha)^{\prime} \in I \text {, for all } \alpha, \beta \in \mathbb{H} \text {. }
$$

Then $\equiv_{I}$ is a congruence relation on $\mathbb{H}$.

Proof. At first, we prove that $\equiv_{I}$ is an equivalence relation on $\mathbb{H}$. Since, for all $\alpha \in \mathbb{H}, \alpha \rightarrow \alpha=1$ and $I$ is a co-filter of $\mathbb{H},(\alpha \rightarrow \alpha)^{\prime}=0 \in I$. Thus, $\alpha \equiv_{I} \alpha$, and so $\equiv_{I}$ is reflexive. It is obvious that $\equiv_{I}$ is symmetric. For proving transitivity of $\equiv_{I}$, suppose $\alpha, \beta, \gamma \in \mathbb{H}$ such that $\alpha \equiv_{I} \beta$ and $\beta \equiv_{I} \gamma$. Hence, $(\alpha \rightarrow \beta)^{\prime},(\beta \rightarrow \alpha)^{\prime},(\beta \rightarrow \gamma)^{\prime}$ and $(\gamma \rightarrow \beta)^{\prime} \in I$ and by Proposition 1(vii) and (viii),

$$
(\alpha \rightarrow \gamma)^{\prime} \leq((\alpha \rightarrow \beta) \odot(\beta \rightarrow \gamma))^{\prime} .
$$


By (HP3) and Propositions 1(vii),(viii) and 2(iii), we have,

$$
\begin{aligned}
\left(\left((\alpha \rightarrow \beta) \rightarrow(\beta \rightarrow \gamma)^{\prime}\right) \rightarrow(\alpha \rightarrow \beta)^{\prime}\right)^{\prime} & =\left(\left((\alpha \rightarrow \beta) \rightarrow(\beta \rightarrow \gamma)^{\prime}\right) \rightarrow((\alpha \rightarrow \beta) \rightarrow 0)\right)^{\prime} \\
& =\left(\left[(\alpha \rightarrow \beta) \odot\left((\alpha \rightarrow \beta) \rightarrow(\beta \rightarrow \gamma)^{\prime}\right)\right] \rightarrow 0\right)^{\prime} \\
& \leq(\beta \rightarrow \gamma)^{\prime \prime \prime} \\
& =(\beta \rightarrow \gamma)^{\prime} \in I .
\end{aligned}
$$

Thus, by Proposition 6(i),

$$
\left(\left((\alpha \rightarrow \beta) \rightarrow(\beta \rightarrow \gamma)^{\prime}\right) \rightarrow(\alpha \rightarrow \beta)^{\prime}\right)^{\prime} \in I
$$

Since $(\alpha \rightarrow \beta)^{\prime} \in I$ and $I$ is a co-filter of $\mathbb{H},(\alpha \rightarrow \beta) \rightarrow(\beta \rightarrow \gamma)^{\prime} \in I$. Moreover, $(\alpha \rightarrow \gamma)^{\prime} \leq(\alpha \rightarrow$ $\beta) \rightarrow(\beta \rightarrow \gamma)^{\prime}$ and $(\alpha \rightarrow \beta) \rightarrow(\beta \rightarrow \gamma)^{\prime} \in I$, by Proposition 6(i), $(\alpha \rightarrow \gamma)^{\prime} \in I$. By the similar way, $(\gamma \rightarrow \alpha)^{\prime} \in I$. Hence, $\alpha \equiv_{I} \gamma$. Therefore, $\equiv_{I}$ is an equivalence relation on $\mathbb{H}$. Now, let $\alpha \equiv_{I} \beta$, for some $\alpha, \beta \in \mathbb{H}$. Then $(\alpha \rightarrow \beta)^{\prime},(\beta \rightarrow \alpha)^{\prime} \in I$. Thus, by Proposition 1 (vi), $\alpha \leq(\alpha \rightarrow \gamma) \rightarrow \gamma$, for all $\gamma \in \mathbb{H}$. So, by Proposition 1(viii), $\beta \rightarrow \alpha \leq \beta \rightarrow((\alpha \rightarrow \gamma) \rightarrow \gamma)$. Then by Proposition 1(viii) and (HP3),

$$
((\alpha \rightarrow \gamma) \rightarrow(\beta \rightarrow \gamma))^{\prime} \leq(\beta \rightarrow \alpha)^{\prime} .
$$

Since $(\beta \rightarrow \alpha)^{\prime} \in I$ and $I$ is a co-filter of $\mathbb{H}$, by Proposition 6(i), $((\alpha \rightarrow \gamma) \rightarrow(\beta \rightarrow \gamma))^{\prime} \in I$. By the similar way, $((\beta \rightarrow \gamma) \rightarrow(\alpha \rightarrow \gamma))^{\prime} \in I$. Hence, $\alpha \rightarrow \gamma \equiv_{I} \beta \rightarrow \gamma$. Suppose $\alpha \equiv_{I} \beta$, for some $\alpha, \beta \in \mathbb{H}$. Then $(\alpha \rightarrow \beta)^{\prime},(\beta \rightarrow \alpha)^{\prime} \in I$ and by Proposition 1(vii) and (HP3), $\alpha \rightarrow \beta \leq(\gamma \rightarrow \alpha) \rightarrow(\gamma \rightarrow \beta)$, for all $\gamma \in \mathbb{H}$. Also, by Proposition 1(viii),

$$
((\gamma \rightarrow \alpha) \rightarrow(\gamma \rightarrow \beta))^{\prime} \leq(\alpha \rightarrow \beta)^{\prime} .
$$

From $(\alpha \rightarrow \beta)^{\prime} \in I$ and $I$ is a co-filter of $\mathbb{H}$, by Proposition 6(i), $((\gamma \rightarrow \alpha) \rightarrow(\gamma \rightarrow \beta))^{\prime} \in I$. By the similar way, $((\gamma \rightarrow \beta) \rightarrow(\gamma \rightarrow \alpha))^{\prime} \in I$. Hence, $\gamma \rightarrow \alpha \equiv_{I} \gamma \rightarrow \beta$. Finally, if $\alpha \equiv_{I} \beta$, for some $\alpha, \beta \in \mathbb{H}$, then $(\alpha \rightarrow \beta)^{\prime},(\beta \rightarrow \alpha)^{\prime} \in I$. From $\alpha \odot \gamma \leq \alpha \odot \gamma$, by Proposition 1(ii),(viii) and (HP3), $\alpha \leq \gamma \rightarrow(\alpha \odot \gamma)$, and so

$$
\beta \rightarrow \alpha \leq \beta \rightarrow(\gamma \rightarrow(\alpha \odot \gamma))=(\beta \odot \gamma) \rightarrow(\alpha \odot \gamma)
$$

Then by Proposition 1(viii),

$$
((\beta \odot \gamma) \rightarrow(\alpha \odot \gamma))^{\prime} \leq(\beta \rightarrow \alpha)^{\prime} .
$$

Since $(\beta \rightarrow \alpha)^{\prime} \in I$ and $I$ is a co-filter of $\mathbb{H}$, by Proposition 6(i), $((\beta \odot \gamma) \rightarrow(\alpha \odot \gamma))^{\prime} \in I$. Similarly, $((\alpha \odot \gamma) \rightarrow(\beta \odot \gamma))^{\prime} \in I$. Hence, $\alpha \odot \gamma \equiv_{I} \beta \odot \gamma$. Therefore, $\equiv_{I}$ is a congruence relation on $\mathbb{H}$.

For any $\alpha \in \mathbb{H}, I_{\alpha}$ will denote the equivalence class of $\alpha$ with respect to $\equiv_{I}$. It is clear that

$$
I_{\alpha}=\left\{\beta \in \mathbb{H} \mid \alpha \equiv_{I} \beta\right\}=\left\{\beta \in \mathbb{H} \mid(\alpha \rightarrow \beta)^{\prime} \in I \text { and }(\beta \rightarrow \alpha)^{\prime} \in I\right\} .
$$

Easily we can see that $I_{0}=I$ and $I_{1}=\left\{\beta \in \mathbb{H} \mid \beta^{\prime} \in I\right\}$.

Theorem 4. Let $\mathbb{H} / I=\left\{I_{\alpha} \mid \alpha \in \mathbb{H}\right\}$. Define the operations $\otimes$ and $\rightsquigarrow$ on $\mathbb{H} /$ I as follows:

$$
I_{\alpha} \otimes I_{\beta}=I_{\alpha \odot \beta} \text { and } I_{\alpha} \rightsquigarrow I_{\beta}=I_{\alpha \rightarrow \beta} .
$$

Then $\left(\mathbb{H} / I, \otimes, \rightsquigarrow, I_{0}, I_{1}\right)$ is a bounded hoop.

Proof. The proof is straightforward. 
Note. Let $\alpha, \beta \in \mathbb{H}$. Then the binary relation " $\leq_{I}$ " is defined on $\mathbb{H} / I$ as follows,

$$
I_{\alpha} \leq_{I} I_{\beta} \text { iff }(\alpha \rightarrow \beta)^{\prime} \in I
$$

Then $\leq_{I}$ is a partially order relation on $\mathbb{H} / I$. Since $(\alpha \rightarrow \alpha)^{\prime}=0 \in I$, for any $\alpha \in \mathbb{H}, I_{\alpha} \leq_{I} I_{\alpha}$. Suppose $I_{\alpha} \leq_{I} I_{\beta}$ and $I_{\beta} \leq_{I} I_{\alpha}$, for any $\alpha, \beta \in \mathbb{H}$. Then $(\alpha \rightarrow \beta)^{\prime} \in I$ and $(\beta \rightarrow \alpha)^{\prime} \in I$. Thus, $\alpha \equiv_{I} \beta$, and so $I_{\alpha}=I_{\beta}$. Now, let $I_{\alpha} \leq_{I} I_{\beta}$ and $I_{\beta} \leq_{I} I_{\gamma}$. Then $(\alpha \rightarrow \beta)^{\prime} \in I$ and $(\beta \rightarrow \gamma)^{\prime} \in I$. By Proposition 1(vii), for $\alpha, \beta, \gamma \in \mathbb{H}$, we have $(\alpha \rightarrow \beta) \odot(\beta \rightarrow \gamma) \leq \alpha \rightarrow \gamma$. Thus, by Proposition 1(viii) and (HP3),

$$
(\alpha \rightarrow \gamma)^{\prime} \leq((\alpha \rightarrow \beta) \odot(\beta \rightarrow \gamma))^{\prime}
$$

and so

$$
(\alpha \rightarrow \gamma)^{\prime} \leq[((\alpha \rightarrow \beta) \odot(\beta \rightarrow \gamma)) \rightarrow 0]
$$

Thus, $(\alpha \rightarrow \gamma)^{\prime} \leq(\alpha \rightarrow \beta) \rightarrow((\beta \rightarrow \gamma) \rightarrow 0)$. Moreover, by Proposition 1(ii),

$$
\alpha \rightarrow \beta \leq(\alpha \rightarrow \gamma)^{\prime} \rightarrow(\beta \rightarrow \gamma)^{\prime}
$$

Hence, by Proposition 1(viii), we obtain,

$$
\left((\alpha \rightarrow \gamma)^{\prime} \rightarrow(\beta \rightarrow \gamma)^{\prime}\right)^{\prime} \leq(\alpha \rightarrow \beta)^{\prime} \in I
$$

Since $(\alpha \rightarrow \beta)^{\prime} \in I$ and $I$ is a co-filter of $\mathbb{H}$, by Proposition $6(\mathrm{i}),\left((\alpha \rightarrow \gamma)^{\prime} \rightarrow(\beta \rightarrow \gamma)^{\prime}\right)^{\prime} \in I$. Also, $(\beta \rightarrow \gamma)^{\prime} \in I$, then $(\alpha \rightarrow \gamma)^{\prime} \in I$. Hence, $I_{\alpha} \leq I_{\gamma}$. Therefore, $\leq_{I}$ is a partially order relation on $\mathbb{H} / I$. For proving $\left(\mathbb{H} / I, \otimes, \rightsquigarrow, I_{0}, I_{1}\right)$ is a bounded hoop, we have $I_{\alpha}=I_{\beta}$ and $I_{\gamma}=I_{\delta}$ iff $\alpha \equiv_{I} \beta$ and $\gamma \equiv_{I} \delta$. Since $\equiv_{I}$ is a congruence relation on $\mathbb{H}$, so all operations are well-defined. Thus, by routine calculations, we can see that $\left(\mathbb{H} / I, \otimes, I_{1}\right)$ is a commutative monoid and (HP2) holds. Let $I_{\alpha}, I_{\beta}, I_{\gamma} \in \mathbb{H} / I$, for any $\alpha, \beta, \gamma \in \mathbb{H}$. Since $\mathbb{H}$ is a hoop, by (HP3) and (HP4) we have,

$$
\left(I_{\alpha} \otimes I_{\beta}\right) \rightsquigarrow I_{\gamma}=I_{(\alpha \odot \beta)} \rightsquigarrow I_{\gamma}=I_{(\alpha \odot \beta) \rightarrow \gamma}=I_{\alpha \rightarrow(\beta \rightarrow \gamma)}=I_{\alpha} \rightsquigarrow\left(I_{\beta \rightarrow \gamma}\right)=I_{\alpha} \rightsquigarrow\left(I_{\beta} \rightsquigarrow I_{\gamma}\right) .
$$

Also, for any $I_{\alpha}, I_{\beta} \in \mathbb{H} / I$, we get

$$
I_{\alpha} \otimes\left(I_{\alpha} \rightsquigarrow I_{\beta}\right)=I_{\alpha} \otimes\left(I_{\alpha \rightarrow \beta}\right)=I_{\alpha \odot(\alpha \rightarrow \beta)}=I_{\beta \odot(\beta \rightarrow \alpha)}=I_{\beta} \otimes\left(I_{\beta \rightarrow \alpha}\right)=I_{\beta} \otimes\left(I_{\beta} \rightsquigarrow I_{\alpha}\right) .
$$

Therefore, $\left(\mathbb{H} / I, \otimes, \rightsquigarrow, I_{0}, I_{1}\right)$ is a bounded hoop.

Example 5. Let $A$ be the hoop as in Example 3. Then $I=\{0, b, d\}$ is a co-filter of $A$. Thus, by routine calculations, we can see that $[b]=[d]=[0]=\{0, b, d\}$ and $[a]=[c]=[1]=\{a, c, 1\}$. Hence, $\frac{A}{\equiv_{I}}=$ $\{[0],[1]\}$. Therefore, $\frac{A}{\equiv_{I}}$ is a bounded hoop.

Example 6. Let $A$ be the hoop as in Example 2. We can see that $A$ does not have (DNP) property, in general. So by Proposition 5 and Example 2, filter and co-filter are different notions. Then $A$ is a co-filter of $A$ and the quotient is $\frac{A}{A}=\{[1]\}$ that is a hoop algebra. But $F=\{b, 1\}$ is a filter of $A$ and the quotient $\frac{A}{\equiv_{F}}=\{[0],[a],[1]\}$ that is a hoop with (DNP).

\section{Some Applications of Co-Filters}

In this section, we try to investigate under which conditions the quotient structure of this congruence relation will be Brouwerian semilattice, Heyting algebra, Wajsberg hoop, Hilbert algebra and BL-algebra. 
Definition 4 ([11]). A Brouwerian lattice is an algebra $\left(\mathbb{H}, \wedge, \vee, \rightarrow,^{\prime}\right)$ with the lattice infimum $(\wedge)$ and the lattice supremum $(\vee)$ in which two operations " " "and " $\rightarrow$ " are defined by $\alpha^{\prime}=\alpha \rightarrow 0$ and

$$
\alpha \wedge \beta \leq \gamma \text { iff } \alpha \leq \beta \rightarrow \gamma
$$

respectively.

Theorem 5. Let I be a co-filter of $\mathbb{H}$ and for all $\alpha \in \mathbb{H}, \alpha^{2}=\alpha$. Then $\mathbb{H} / \mathrm{I}$ is a Brouwerian semilattice.

Proof. Let $I$ be a co-filter of $\mathbb{H}$. By Theorem $4, \mathbb{H} / I$ is a hoop. Thus, by Proposition $1(\mathrm{i}),\left(\mathbb{H} / I, \leq_{I}\right)$ is a meet-semilattice with $I_{\alpha} \wedge_{I} I_{\beta}=I_{\alpha} \otimes\left(I_{\alpha} \rightsquigarrow I_{\beta}\right)$, for all $I_{\alpha}, I_{\beta} \in \mathbb{H} / I$. Now, we prove that, for all $I_{\alpha}, I_{\beta} \in \mathbb{H} / I$,

$$
I_{\alpha} \wedge_{I} I_{\beta} \leq I_{\gamma} \text { iff } I_{\alpha} \leq I_{\beta} \rightsquigarrow I_{\gamma} .
$$

Since $\mathbb{H} / I$ is a hoop, by Proposition 1(iii), $I_{\alpha} \otimes I_{\beta} \leq I_{\alpha} \wedge_{I} I_{\beta} \leq I_{\gamma}$. Thus, $I_{\alpha} \otimes I_{\beta} \leq I_{\gamma}$, and so by Proposition 1(ii), $I_{\alpha} \leq I_{\beta} \rightsquigarrow I_{\gamma}$. Conversely, suppose $I_{\alpha} \leq I_{\beta} \rightsquigarrow I_{\gamma}$, for all $I_{\alpha}, I_{\beta}, I_{\gamma} \in \mathbb{H} / I$. According to definition of $\leq_{I},(\alpha \rightarrow(\beta \rightarrow \gamma))^{\prime} \in I$. By Proposition 1(vii), $\beta \rightarrow \gamma \leq(\alpha \rightarrow \beta) \rightarrow(\alpha \rightarrow \gamma)$ and by (HP3), $\beta \rightarrow \gamma \leq(\alpha \odot(\alpha \rightarrow \beta)) \rightarrow \gamma$. Also, by Proposition 1(viii) and (HP3), we get

$$
\alpha \rightarrow(\beta \rightarrow \gamma) \leq \alpha \rightarrow((\alpha \odot(\alpha \rightarrow \beta)) \rightarrow \gamma)
$$

and so

$$
\alpha \rightarrow(\beta \rightarrow \gamma) \leq(\alpha \odot(\alpha \odot(\alpha \rightarrow \beta)) \rightarrow \gamma)
$$

Thus,

$$
\alpha \rightarrow(\beta \rightarrow \gamma) \leq(((\alpha \odot \alpha) \odot(\alpha \rightarrow \beta)) \rightarrow \gamma) .
$$

Since for any $\alpha \in \mathbb{H}, \alpha^{2}=\alpha$, we obtain, $\alpha \rightarrow(\beta \rightarrow \gamma) \leq((\alpha \odot(\alpha \rightarrow \beta)) \rightarrow \gamma)$ and so,

$$
\alpha \rightarrow(\beta \rightarrow \gamma) \leq(\alpha \rightarrow \beta) \rightarrow(\alpha \rightarrow \gamma)
$$

Hence, by Proposition 1(viii), we get $((\alpha \rightarrow \beta) \rightarrow(\alpha \rightarrow \gamma))^{\prime} \leq(\alpha \rightarrow(\beta \rightarrow \gamma))^{\prime}$. Since $I$ is a co-filter of $\mathbb{H}$ and $(\alpha \rightarrow(\beta \rightarrow \gamma))^{\prime} \in I$, by Proposition 6(i), $((\alpha \rightarrow \beta) \rightarrow(\alpha \rightarrow \gamma))^{\prime} \in I$, so $I_{\alpha} \rightsquigarrow I_{\beta} \leq I_{\alpha} \rightsquigarrow I_{\gamma}$. Thus, by Proposition 1(ii),(i) and (viii),

$$
I_{\alpha} \wedge_{I} I_{\beta}=I_{\alpha} \otimes\left(I_{\alpha} \rightsquigarrow I_{\beta}\right) \leq I_{\alpha} \otimes\left(I_{\alpha} \rightsquigarrow I_{\gamma}\right)=I_{\alpha} \wedge I_{\gamma} \leq I_{\gamma}
$$

Hence, $I_{\alpha} \wedge_{I} I_{\beta} \leq I_{\gamma}$. Therefore, $\mathbb{H} / I$ is a Brouwerian semilattice.

Example 7. Let $\mathbb{H}=\{0, a, b, c, 1\}$ be a set with two operations which are given below:

\begin{tabular}{l|lllll}
$\rightarrow$ & 0 & $a$ & $b$ & $c$ & 1 \\
\hline 0 & 1 & 1 & 1 & 1 & 1 \\
$a$ & $b$ & 1 & $b$ & 1 & 1 \\
$b$ & $a$ & $a$ & 1 & 1 & 1 \\
$c$ & 0 & $a$ & $b$ & 1 & 1 \\
1 & 0 & $a$ & $b$ & $c$ & 1
\end{tabular}

\begin{tabular}{l|lllll}
$\odot$ & 0 & $a$ & $b$ & $c$ & 1 \\
\hline 0 & 0 & 0 & 0 & 0 & 0 \\
$a$ & 0 & $a$ & 0 & $a$ & $a$ \\
$b$ & 0 & 0 & $b$ & $b$ & $b$ \\
$c$ & 0 & $a$ & $b$ & $c$ & $c$ \\
1 & 0 & $a$ & $b$ & $c$ & 1
\end{tabular}

Thus, $(\mathbb{H}, \odot, \rightarrow, 0,1)$ is a hoop and $\alpha^{2}=\alpha$, for all $\alpha \in \mathbb{H}$. Then $I=\{0, a\}$ is a co-filter of $\mathbb{H}, I_{0}=I_{a}=I$ and $I_{b}=I_{c}=I_{1}=\{b, c, 1\}$. Hence, by Theorem $5, \mathbb{H} / I=\left\{I_{0}, I_{1}\right\}$ is a Brouwerian semilattice.

Theorem 6. Let $\mathbb{H}$ has (DNP) and $\mathbb{H} /$ I be a Brouwerian semilattice. Then I is a co-filter of $\mathbb{H}$. 
Proof. Let $I_{\alpha} \otimes_{I} I_{\beta}=I_{\alpha} \wedge_{I} I_{\beta}$, for all $I_{\alpha}, I_{\beta} \in \mathbb{H} / I$. Then $I_{\alpha} \otimes_{I} I_{\alpha}=I_{\alpha} \wedge_{I} I_{\alpha}=I_{\alpha}$. Thus, $I_{\alpha^{2}}=I_{\alpha}$, and so $\left(\alpha^{2} \rightarrow \alpha\right)^{\prime} \in I$. By Proposition 1(iii), $0 \in I$. Now, suppose $(\alpha \rightarrow \beta)^{\prime}$ and $\beta \in I$, for some $\alpha, \beta \in \mathbb{H}$. Since $I=I_{0}$, we have $\beta \in I_{0}$. It means that $(\beta \rightarrow 0)^{\prime} \in I$, and equivalently $I_{\beta} \leq I_{0}$. Moreover, $(\alpha \rightarrow \beta)^{\prime} \in I$, then $I_{\alpha} \leq I_{\beta}$, and so $I_{\alpha} \leq I_{0}$ i.e., $(\alpha \rightarrow 0)^{\prime} \in I$. Hence, $\alpha^{\prime \prime} \in I$. Since $\mathbb{H}$ has (DNP), we get $\alpha \in I$. Therefore, $I$ is a co-filter of $\mathbb{H}$.

Definition 5 ([11]). A hoop $(\mathbb{H}, \odot, \rightarrow, 1)$ is called Wajsberg if, for any $\alpha, \beta \in \mathbb{H}$,

$$
(\alpha \rightarrow \beta) \rightarrow \beta=(\beta \rightarrow \alpha) \rightarrow \alpha .
$$

Theorem 7. Let $\mathbb{H}$ has (DNP). Then I is a co-filter of $\mathbb{H}$ iff $\mathbb{H} /$ I is a Wajsberg hoop.

Proof. $(\Rightarrow)$ Since $\mathbb{H}$ has (DNP), by Proposition 2(v), $(\alpha \rightarrow \beta) \rightarrow \beta=(\beta \rightarrow \alpha) \rightarrow \alpha$, for all $\alpha, \beta \in \mathbb{H}$. Thus,

$$
(((\alpha \rightarrow \beta) \rightarrow \beta) \rightarrow((\beta \rightarrow \alpha) \rightarrow \alpha))^{\prime}=0 \in I,
$$

and so $\left(I_{\alpha} \rightsquigarrow I_{\beta}\right) \rightsquigarrow I_{\beta} \leq\left(I_{\beta} \rightsquigarrow I_{\alpha}\right) \rightsquigarrow I_{\alpha}$. By the similar way, $\left(I_{\beta} \rightsquigarrow I_{\alpha}\right) \rightsquigarrow I_{\alpha} \leq\left(I_{\alpha} \rightsquigarrow I_{\beta}\right) \rightsquigarrow I_{\beta}$. Thus, $\left(I_{\alpha} \rightsquigarrow I_{\beta}\right) \rightsquigarrow I_{\beta}=\left(I_{\beta} \rightsquigarrow I_{\alpha}\right) \rightsquigarrow I_{\alpha}$, for all $I_{\alpha}, I_{\beta} \in \mathbb{H} / I$. Therefore, $\mathbb{H} / I$ is a Wajsberg hoop.

$(\Leftarrow)$ The proof is similar to the proof of Theorem 6 .

Example 8. In Example $1, \mathbb{H}$ is a hoop with (DNP). Since $I=\{0, b, d\}$ is a co-filter of $\mathbb{H}, I_{0}=I_{b}=I_{d}=I$ and $I_{a}=I_{c}=I_{1}=\{a, c, 1\}$. Hence, by Theorem $7, \mathbb{H} / I=\left\{I_{0}, I_{1}\right\}$ is a Wajsberg hoop.

Definition 6 ([11]). A Heyting algebra is an algebra $(A, \vee, \wedge, \rightarrow, 1)$, where $(A, \vee, \wedge, 1)$ is a distributive lattice with the greatest element and the binary operation $\rightarrow$ on $A$ verifies, for any $x, y, z \in A$,

$$
x \leq y \rightarrow z \text { iff } x \wedge y \leq z .
$$

Theorem 8. Let $\mathbb{H}$ has (DNP) and $\alpha^{2}=\alpha$, for all $\alpha \in \mathbb{H}$. Then I is a co-filter of $\mathbb{H}$ iff $\mathbb{H} /$ I is a Heyting algebra.

Proof. $(\Rightarrow)$ Since $I$ is a co-filter of $\mathbb{H}$ and $\alpha^{2}=\alpha$, for all $\alpha \in \mathbb{H}$, by Theorem $5, \mathbb{H} / I$ is a Brouwerian semilattice. Moreover, since $\mathbb{H}$ has (DNP), by Theorem $7, \mathbb{H} / I$ is a Wajsberg hoop. Define $I_{\alpha} \vee_{I} I_{\beta}=$ $\left(I_{\beta} \rightsquigarrow I_{\alpha}\right) \rightsquigarrow I_{\alpha}$, for all $I_{\alpha}, I_{\beta} \in \mathbb{H} / I$. Then by Propositions 3 and $4,\left(\mathbb{H} / I, \wedge_{I}, \vee_{I}\right)$ is a distributive lattice. Therefore, $\mathbb{H} / I$ is a Heyting algebra.

$(\Leftarrow)$ Since $\mathbb{H} / I$ is a Heyting algebra, it is a Brouwerian semilattice. On the other side, $\mathbb{H}$ has (DNP), then by Theorem 6, I is a co-filter of $\mathbb{H}$.

Example 9. Let $\mathbb{H}=\{0, a, b, 1\}$ be a set with the following Cayley tabels,

\begin{tabular}{l|llll}
$\rightarrow$ & 0 & $a$ & $b$ & 1 \\
\hline 0 & 1 & 1 & 1 & 1 \\
$a$ & $b$ & 1 & $b$ & 1 \\
$b$ & $a$ & $a$ & 1 & 1 \\
1 & 0 & $a$ & $b$ & 1
\end{tabular}

\begin{tabular}{c|cccc}
$\odot$ & 0 & $a$ & $b$ & 1 \\
\hline 0 & 0 & 0 & 0 & 0 \\
$a$ & 0 & $a$ & 0 & $a$ \\
$b$ & 0 & 0 & $b$ & $b$ \\
1 & 0 & $a$ & $b$ & 1
\end{tabular}

Then $(\mathbb{H}, \odot, \rightarrow, 0,1)$ is a hoop with (DNP) and for any $\alpha \in \mathbb{H}, \alpha^{2}=\alpha$. From $I=\{0, b\}$ is a co-filter of $\mathbb{H}$, $I_{0}=I_{b}=I$ and $I_{1}=I_{a}=\{1, a\}$. Then by Theorem $8, \mathbb{H} / I=\left\{I, I_{1}\right\}$ is a Heyting algebra.

Definition 7 ([11]). A Hilbert algebra is a tripe $(A, \rightarrow, 1)$ of type $(2,0)$ such that, for all $x, y, z \in A$, the following three axioms are satisfied,

$(H 1) x \rightarrow(y \rightarrow x)=1$. 
(H2) $x \rightarrow(y \rightarrow z)=(x \rightarrow y) \rightarrow(x \rightarrow z)$.

(H3) If $x \rightarrow y=y \rightarrow x=1$, then $x=y$.

The Hilbert algebra induces a partial order $\leq$ on $A$, defined by, $x \leq y$ iff $x \rightarrow y=1$ and 1 is the greatest element of the induced poset $(A, \leq)$. A Hilbert algebra $A$ is bounded if there is an element $0 \in A$ such that, for any $x \in A, 0 \leq x$.

Lemma 1. Let $\alpha^{2}=\alpha$, for all $\alpha \in \mathbb{H}$. Then, for all $\alpha, \beta, \gamma \in \mathbb{H}$,

$$
\alpha \rightarrow(\beta \rightarrow \gamma)=(\alpha \rightarrow \beta) \rightarrow(\alpha \rightarrow \gamma)
$$

Proof. Let $\alpha \in \mathbb{H}$ such that $\alpha^{2}=\alpha$. Then by Proposition 1 (iv), $\beta \leq \alpha \rightarrow \beta$, for any $\alpha, \beta \in \mathbb{H}$ and by Proposition 1(viii), $\alpha \rightarrow((\alpha \rightarrow \beta) \rightarrow \gamma) \leq \alpha \rightarrow(\beta \rightarrow \gamma)$. Then by (HP3), $(\alpha \rightarrow \beta) \rightarrow(\alpha \rightarrow \gamma) \leq \alpha \rightarrow$ $(\beta \rightarrow \gamma)$. Conversely, by (HP3), for all $\alpha, \beta, \gamma \in \mathbb{H}$,

$$
[\alpha \rightarrow(\beta \rightarrow \gamma)] \rightarrow[(\alpha \rightarrow \beta) \rightarrow(\alpha \rightarrow \gamma)]=[(\alpha \rightarrow \beta) \odot \alpha \odot(\alpha \rightarrow(\beta \rightarrow \gamma))] \rightarrow \gamma .
$$

By Proposition 1(vii), $\alpha \odot(\alpha \rightarrow(\beta \rightarrow \gamma)) \leq \beta \rightarrow \gamma$. Then by Proposition 1(viii) and (vii),

$$
(\alpha \rightarrow \beta) \odot \alpha \odot(\alpha \rightarrow(\beta \rightarrow \gamma)) \leq(\alpha \rightarrow \beta) \odot(\beta \rightarrow \gamma) \leq \alpha \rightarrow \gamma .
$$

Thus, $\alpha^{2} \odot(\alpha \rightarrow \beta) \odot(\alpha \rightarrow(\beta \rightarrow \gamma)) \leq \gamma$. Since $\alpha^{2}=\alpha$, we get $\alpha \odot(\alpha \rightarrow \beta) \odot(\alpha \rightarrow(\beta \rightarrow \gamma)) \leq \gamma$. Hence, by (HP3), $\alpha \rightarrow(\beta \rightarrow \gamma) \leq(\alpha \rightarrow \beta) \rightarrow(\alpha \rightarrow \gamma)$.

Theorem 9. Let I be a co-filter of $\mathbb{H}$ and $\alpha^{2}=\alpha$, for all $\alpha \in \mathbb{H}$. Then $\mathbb{H} /$ I is a Hilbert algebra.

Proof. Since $I$ is a co-filter of $\mathbb{H}$, by Theorem $5, \mathbb{H} / I$ is a hoop. Thus by Proposition 1 (iv), it is clear that $I_{\alpha} \rightsquigarrow\left(I_{\beta} \rightsquigarrow I_{\alpha}\right)=I_{1}$, for all $I_{\alpha}, I_{\beta} \in \mathbb{H} / I$. Let $I_{\alpha}, I_{\beta} \in \mathbb{H} / I$ such that $I_{\alpha} \rightsquigarrow I_{\beta}=I_{\beta} \rightsquigarrow I_{\alpha}=I_{1}$. Then $(\alpha \rightarrow \beta)^{\prime} \in I$ and $(\beta \rightarrow \alpha)^{\prime} \in I$ and so $\alpha \equiv_{I} \beta$. Hence, $I_{\alpha}=I_{\beta}$. Moreover, since $\alpha^{2}=\alpha$, for all $\alpha \in \mathbb{H}$, by Lemma $1, \alpha \rightarrow(\beta \rightarrow \gamma)=(\alpha \rightarrow \beta) \rightarrow(\alpha \rightarrow \gamma)$, for all $\alpha, \beta, \gamma \in \mathbb{H}$, and so

$$
[(\alpha \rightarrow(\beta \rightarrow \gamma)) \rightarrow((\alpha \rightarrow \beta) \rightarrow(\alpha \rightarrow \gamma))]^{\prime}=0 \in I .
$$

Thus, by definition of $I_{1}$,

$$
\left(I_{\alpha} \rightsquigarrow\left(I_{\beta} \rightsquigarrow I_{\gamma}\right)\right) \rightsquigarrow\left(\left(I_{\alpha} \rightsquigarrow I_{\beta}\right) \rightsquigarrow\left(I_{\alpha} \rightsquigarrow I_{\gamma}\right)\right)=I_{1}
$$

Therefore, $\mathbb{H} / I$ is a Hilbert algebra.

Definition 8 ([11]). A BL-algebra is an algebra $(A, \vee, \wedge, \odot, \rightarrow, 0,1)$ of type $(2,2,2,2,0,0)$ that, for any $x, y, z \in A$, it is satisfying the following axioms:

(BL1) $(A, \vee, \wedge, 0,1)$ is a bounded lattice.

(BL2) $(A, \odot, 1)$ is a commutative monoid.

(BL3) $x \odot y \leq z$ iff $x \leq y \rightarrow z$.

$(B L 4)(x \rightarrow y) \vee(y \rightarrow x)=1$.

$(B L 5) x \wedge y=x \odot(x \rightarrow y)$.

Theorem 10. Let $\mathbb{H}$ be a $\vee$-hoop such that, for all $\alpha \in \mathbb{H}, \alpha^{2}=\alpha$ and I be a co-filter of $\mathbb{H}$. Then $\mathbb{H} / I$ is $a$ BL-algebra.

Proof. Let $\mathbb{H}$ be a $\vee$-hoop. Then $\mathbb{H} / I$ is a $\vee_{I}$-hoop. Thus, by Proposition $4,\left(\mathbb{H} / I, \wedge_{I}, \vee_{I}, I_{0}, I_{1}\right)$ is a bounded distributive lattice. Now, we prove that $\mathbb{H} / I$ is a BL-algebra. For this, it is enough to prove that 
$\left(I_{\alpha} \rightsquigarrow I_{\beta}\right) \vee_{I}\left(I_{\beta} \rightsquigarrow I_{\alpha}\right)=I_{1}$, for all $I_{\alpha}, I_{\beta} \in \mathbb{H} / I$. Equivalently, we show that $((\alpha \rightarrow \beta) \vee(\beta \rightarrow \alpha))^{\prime} \in I$, for all $\alpha, \beta \in \mathbb{H}$. Since for all $\alpha, \beta \in \mathbb{H}$,

$$
(\alpha \rightarrow \beta),(\beta \rightarrow \alpha) \leq(\alpha \rightarrow \beta) \vee(\beta \rightarrow \alpha),
$$

by Proposition 1(viii),

$$
((\alpha \rightarrow \beta) \vee(\beta \rightarrow \alpha))^{\prime} \leq(\alpha \rightarrow \beta)^{\prime} \wedge(\beta \rightarrow \alpha)^{\prime} .
$$

On the other hand, by Proposition 1(iv), $\beta \leq \alpha \rightarrow \beta$ and by Proposition 2(ii), $\beta^{\prime} \leq \beta \rightarrow \alpha$, then by Proposition 1(viii), $(\alpha \rightarrow \beta)^{\prime} \leq \beta^{\prime}$ and $(\beta \rightarrow \alpha)^{\prime} \leq \beta^{\prime \prime}$. Thus, by Propositions 1(i), 2(i) and $\alpha^{2}=\alpha$, for all $\alpha \in \mathbb{H}$, we have

$$
\begin{aligned}
((\alpha \rightarrow \beta) \vee(\beta \rightarrow \alpha))^{\prime} & \leq(\alpha \rightarrow \beta)^{\prime} \wedge(\beta \rightarrow \alpha)^{\prime} \\
& \leq \beta^{\prime} \wedge \beta^{\prime \prime} \\
& =\beta^{\prime} \odot\left(\beta^{\prime} \rightarrow \beta^{\prime \prime}\right) \quad \text { by Proposition } 1(i) \\
& =\beta^{\prime} \odot\left(\beta^{\prime} \rightarrow\left(\beta^{\prime} \rightarrow 0\right)\right) \quad \text { by }(\mathrm{HP} 3) \\
& =\beta^{\prime} \odot\left(\left(\beta^{\prime}\right)^{2} \rightarrow 0\right) \quad \text { by } \alpha^{2}=\alpha \\
& =\beta^{\prime} \odot\left(\beta^{\prime} \rightarrow 0\right) \quad \text { by Proposition } 2(i) \\
& =\beta^{\prime} \odot \beta^{\prime \prime} \\
& =0 .
\end{aligned}
$$

Then $((\alpha \rightarrow \beta) \vee(\beta \rightarrow \alpha))^{\prime}=0 \in I$. Therefore, $\mathbb{H} / I$ is a BL-algebra.

Theorem 11. Let $\mathbb{H}$ has (DNP) and $\alpha^{2}=\alpha$, for all $\alpha \in \mathbb{H}$. Then I is a co-filter of $\mathbb{H}$ iff $\mathbb{H} / I$ is a BL-algebra.

Proof. $(\Rightarrow)$ Since $\mathbb{H}$ has $(\mathrm{DNP})$ and $I$ is a co-filter of $\mathbb{H}$, by Theorem $7, \mathbb{H} / I$ is a Wajsberg hoop. Define $I_{\alpha} \vee_{I} I_{\beta}=\left(I_{\alpha} \rightsquigarrow I_{\beta}\right) \rightsquigarrow I_{\beta}$ for all $I_{\alpha}, I_{\beta} \in \mathbb{H} / I$. Then by Proposition $3, \mathbb{H} / I$ is a $\vee_{I}$-hoop, and so by Proposition $4,\left(\mathbb{H} / I, \wedge_{I}, \vee_{I}, I_{0}, I_{1}\right)$ is a bounded lattice. On the other side, since $\alpha^{2}=\alpha$, for all $\alpha \in \mathbb{H}$, by Theorem $10, \mathbb{H} / I$ is a BL-algebra.

$(\Leftarrow)$ Since $\mathbb{H}$ has $(\mathrm{DNP})$ and $\mathbb{H} / I$ is a BL-algebra, $\mathbb{H} / I$ is a distributive lattice. Thus, by Theorem $6, I$ is a co-filter of $\mathbb{H}$.

Remark 1. As you see in this section, we investigated the relation among the quotient hoop $\frac{A}{T}$ that is made by a co-filter I with other logical algebras such as Brouwerian semi-lattice, Heyting algebra, Hilbert algebra, Wajsberg hoop and BL-algebra. Clearly these conditions are similar and we know that for example if $A$ has Godel condition $\left(x^{2}=x\right)$ then $\frac{A}{I}$ is Hilbert algebra and by adding $(D N P)$ property to $A$ we obtain that $\frac{A}{I}$ is Heyting algebra.

\section{Conclusions and Future Works}

We have introduced the notion of co-filter of hoops and a congruence relation on hoop, and then we have constructed the quotient structures by using co-filters. We have considered the relation between filters and co-filters in a hoop with (DNP) property. We have provided conditions for a subset to be a co-filter. We have discussed characterizations of a co-filter. We have studied the relation among this structure and other algebraic structures. Using the notion of co-filters, we have established the quotient Brouwerian semilattice, the quotient Hilbert algebra and the quotient BL-algebra. We have induced a co-filter from a quotient Brouwerian semilattice. In our subsequent research, we will study some kinds of co-filter such as, implicative, ultra and prime one and investigate the relation between them. Also, we will discuss fuzzy co-filters and fuzzy congruence relation by them and study the quotient structure of this fuzzy congruence relation.

Author Contributions: Creation and Mathematical Ideas, R.A.B.; writing-original draft preparation, M.A.K.; writing-review and editing, S.-Z.S. and Y.B.J.; funding acquisition, S.-Z.S. 
Funding: This research was supported by Basic Science Research Program through the National Research Foundation of Korea(NRF) funded by the Ministry of Education(No. 2016R1D1A1B02006812).

Acknowledgments: The authors express their sincere gratitude to the unknown reviewers for their detailed reading and valuable advice.

Conflicts of Interest: The authors declare no conflict of interest.

\section{References}

1. Bosbach, B. Komplementäre Halbgruppen. Kongruenzen und Quatienten. Funda. Math. 1970, 69, 1-14. [CrossRef]

2. Bosbach, B. Komplementäre Halbgruppen. Axiomatik und Arithmetik. Funda. Math. 1969, 64, $257-287$. [CrossRef]

3. Aaly Kologani, M.; Borzooei, R.A. On ideal theory of hoops. Math. Bohem. 2019. [CrossRef]

4. Borzooei, R.A.; Aaly kologani, M. Filter theory of hoop-algebras. J. Adv. Res. Pure Math. 2014, 6, 1-15. [CrossRef]

5. Alavi, S.Z.; Borzooei, R.A.; Aaly Kologani, M. Filter theory of pseudo hoop-algebras. Ital. J. Pure Appl. Math. 2017, 37, 619-632.

6. Namdar, A.; Borzooei, R.A. Nodal filters in hoop algebras. Soft Comput. 2018, 22, 7119-7128. [CrossRef]

7. Namdar, A.; Borzooei, R.A.; Borumand Saeid, A.; Aaly Kologani, M. Some results in hoop algebras. J. Intell. Fuzzy Syst. 2017, 32, 1805-1813. [CrossRef]

8. Rezaei, A.; Borumand Saeid, A.; Borzooei, R.A. Some types of filters in BE-algebras. Math. Comput. Sci. 2013, 7, 341-352.

9. Aglianò, P.; Ferreirim, I.M.A.; Montagna, F. Basic hoops: An Algebraic Study of Continuous T-Norms. Stud. Log. 2007, 87, 73-98. [CrossRef]

10. Georgescu, G.; Leustean, L.; Preoteasa, V. Pseudo-hoops. J. Mult.-Valued Log. Soft Comput. 2005, 11, $153-184$.

11. Iorgulescu, A. Algebras of logic as BCK-algebras; Editura ASE: Bucharest, Romania, 2008.

(C) 2019 by the authors. Licensee MDPI, Basel, Switzerland. This article is an open access article distributed under the terms and conditions of the Creative Commons Attribution (CC BY) license (http://creativecommons.org/licenses/by/4.0/). 